\title{
Liderazgo en el gobierno universitario e innovaciones en la docencia: una revisión de la literatura
}

Emilio Rodríguez-Ponce, Liliana Pedraja-Rejas y Julio Labraña

\section{RESUMEN}

Este artículo ${ }^{1}$ examina la relación entre estilos de liderazgo en el gobierno universitario y la calidad de la docencia universitaria. El artículo se divide en cuatro apartados. Primero, examinamos la relevancia de los estilos de liderazgo en las universidades. A continuación, exploramos la evidencia sobre la relación entre liderazgo e innovaciones docentes, subrayando las ventajas de los estilos de liderazgos transformacionales y distribuidos. La tercera sección discute esta evidencia en el contexto de los sistemas latinoamericanos, elaborando un modelo útil para la investigación y la gestión de las universidades. El ensayo termina con un resumen, líneas de investigación y sugerencias para la gestión de la enseñanza.

Palabras clave: educación superior, estilos de liderazgo, docencia universitaria.

Emilio Rodríguez-Ponce

Chileno. Doctor en Dirección de Empresas, Universidad Complutense de Madrid, España; Doctor en Educación, Universidad Autónoma de Barcelona, España; Magíster en Administración mención Finanzas, Universidad de Chile. Profesor Titular del Instituto de Alta Investigación de la Universidad de Tarapacá, Chile. Temas de investigación: educación superior; calidad y acreditación, liderazgo educativo y formación inicial docente. ORCID: https://orcid.org/0000-0003-4861-002X.

\section{Liliana Pedraja-Rejas}

Ipedraja@uta.cl

Chilena. Doctora en Administración y Dirección de Empresas, Universidad Politécnica de Valencia, España; Doctora en Ciencias de la Educación, Pontificia Universidad Católica de Chile; Master Universitario en Dirección y Marketing, y Magíster en Educación. Profesora Titular del Departamento de Ingeniería Industrial y de Sistemas, Facultad de Ingeniería, Universidad de Tarapacá, Chile. Temas de investigación: calidad, liderazgo, gestión institucional y formación inicial docente. ORCID: https://orcid.org/0000-0001$7732-4183$.

\section{Julio Labraña}

juliolabranavargas@gmail.com

Chileno. Doctor en Sociología, Universidad Witten/Herdecke, Alemania; Magíster en Análisis Sistémico aplicado a la Sociedad, Universidad de Chile; Sociólogo, Universidad de Concepción, Chile. Investigador asociado del Centro Comparado de Políticas Públicas de la Universidad Diego Portales, Chile. Temas de investigación: sociología de la educación, sistemas de educación superior, teoría de sistemas sociales e innovación en la educación superior. ORCID: https://orcid.org/0000-0003-2441-8260.

${ }^{1}$ Texto elaborado en el marcoy con el apoyo delProyecto FONDECyT No 1180484 . "Determinantes, procesos y resultados de las carreras de educación parvularia y pedagogía en educación básica. Elementos críticos e imperativos estratégicos". 


\section{Liderança no governo universitário e inovações na docência: uma revisão da literatura}

\section{RESUMO}

Este artigo examina a relação entre estilos de lideranças no governo universitário e a qualidade da docência universitária. $\mathrm{O}$ artigo se divide em quatro partes. Primeiro, examinamos a relevância dos estilos de liderança nas universidades. A continuação, pesquisamos a evidência sobre a relação entre liderança e inovações docentes, destacando as vantagens dos estilos de liderança transformativos e distribuídos. A terceira seção discute esta evidência no contexto dos sistemas latino-americanos, elaborando um modelo útil para a pesquisa e a gestão das universidades. O ensaio termina com um resumo, linhas de pesquisa e sugestões para a gestão do ensino.

Palavras chave: educação superior, estilos de liderança, docência universitária.

\section{University governance leadership and teaching innovations: a review of the literature}

\section{ABSTRACT}

This article examines the relationship between leadership styles in university governance and the quality of university teaching. The article is divided into four sections. First, the authors examine the relevance of leadership styles in universities. Next, they explore the evidence about the relationship between leadership and teaching innovations, highlighting the advantages of transformational and distributed leadership styles. The third section discusses this evidence in the context of Latin American systems, elaborating a useful model for university research and management. The essay ends with a summary, lines of research and suggestions for teaching management.

Key words: higher education, leadership styles, university teaching. 


\section{Introducción}

La docencia universitaria se ha debido enfrentar a desafios considerables durante las últimas décadas. Tres desarrollos nos parecen en este sentido especialmente significativos. En primer lugar, la ampliación del acceso a los sistemas de educación superior, representado en el paso de sistemas de élite a sistemas masificados y luego de acceso universal (Trow, 2007; Labraña y Vanderstraeten, 2020). Este desarrollo ha producido una mayor heterogeneidad en las características académicas de los estudiantes, que ahora no sólo provienen de las clases sociales superiores, como ocurría en décadas anteriores, sino que proceden de diferentes grupos sociales (Leach, 2013; Kosunen, 2018). Como resultado, la docencia a nivel universitario debe hoy lidiar con estudiantes con múltiples necesidades, impulsando el establecimiento de sistemas internos que les permitan responder de manera individualizada a las demandas de los distintos alumnos y de este modo impulsar el aprovechamiento de su paso por la universidad (Hemmati y Mahdie, 2020).

En una dirección similar, la creciente relevancia de las competencias adquiridas por los estudiantes en las universidades en el marco de la emergencia de una economía de la información y del conocimiento obliga igualmente a estas instituciones a prestar mayor atención a la estructuración de procesos de enseñanza y aprendizaje y sus impactos en la formación (Steinbicker, 2011). En efecto, no se trata ya simplemente de la adquisición de una serie de conocimientos durante un periodo acotado de la vida, sino de la interiorización de habilidades generales, tanto técnicas como cognitivas, emocionales y sociales, de manera continua por parte de los estudiantes, que faciliten una vez egresados su inserción en mercados laborales cambiantes y, de ser necesario, su reconversión para incorporarse en distintos tipos de empleos (World Bank, 2003; Schwab, 2016).

Finalmente, y no menos importante, los sistemas de educación superior a nivel global han experimentado también transformaciones importantes en lo que respecta a su financiamiento, las cuales también han alterado sus dinámicas docentes (Kivistö y Kohtamäki, 2016; Feixas, Martinez-Usarralde y López-Martín, 2018). En este sentido, el monto del presupuesto de las universidades que proviene de fondos estatales ha tendido a reducirse, aumentando significativamente la importancia de los ingresos por concepto de matrícula, lo que ha repercutido en la concepción de los estudiantes como consumidores (Molesworth, Nixon y Scullion, 2009) y una mayor atención de las universidades a la gestión de sus actividades docentes (Zepke, 2015), de modo tal que estas pueden presentarse en un mercado crecientemente competitivo como una alternativa formativa de calidad para los estudiantes y sus familias (Brunner et al., 2021).

La combinación de estos tres desarrollos - masificación, relevancia de competencias en la economía de la información y el conocimiento, y aumento de la importancia de recursos por concepto de matrícula - resultan en una considerable presión para que las instituciones de educación superior mejoren sus procesos de enseñanza y aprendizaje. La capacidad de innovación, esto es, crear nuevas ideas, productos y procesos que puedan ser empleados para elevar la calidad de los procesos pedagógicos (López Martin, 2017; Alonso-Sáez, Darretxe y Beloki, 2019), se convierte en un valor central a nivel organizacional, en tanto hace posible la búsqueda y experimentación con soluciones alternativas ante circunstancias cambiantes y así seleccionar, entre las nuevas opciones identificadas, aquellas que demuestren su utilidad en términos de aprendizaje de los estudiantes e incorporarlas en del repertorio docente de los académicos de la universidad.

En esta perspectiva, como muestra la literatura especializada, la innovación no depende sólo de los atributos individuales de las personas, sino también de la capacidad de las instituciones de asegurar la existencia de espacios organizacionales receptivos a la generación, experimentación e institucionalización de nuevas ideas (Mendoza, 2015). Aplicando este principio 
a las universidades, no resulta suficiente por tanto con la mera creatividad individual de los docentes para innovar en sus metodologías de enseñanza, sino que se requiere de una estructura organizacional que permita que aquellas experiencias innovadoras, que efectivamente hayan permitido mejorar la calidad de la enseñanza, sean reconocidas y generalizadas a lo largo de la institución, de modo tal que otros docentes, enfrentados a situaciones semejantes, puedan también hacer uso de estos conocimientos.

El liderazgo a nivel de gobierno universitario resulta fundamental en este contexto, en tanto contribuye a sentar las bases para la existencia de una estructura organizacional de este tipo. Sin embargo, a pesar de su importancia, este tema no parece ser un tópico sistemáticamente estudiado en la investigación en educación superior. A modo de ilustración, la búsqueda de "liderazgo", "docencia" e "innovación" en educación superior en Web of Science, el repositorio científico más prestigioso, arroja apenas 47 resultados, con la mayoría de los estudios enfocados en las competencias docentes de los académicos (Al-Husseini y Elbeltagi, 2016; Sutton y DeSantis, 2017; Fraser, 2019; Hattam y Weiler, 2021).

El presente ensayo se propone avanzar en una dirección complementaria a la del creciente número de estudios en liderazgo y universidad en América Latina (Ordorika, 1995; Ordorika, Martínez y Ramírez, 2011; Ganga et al., 2018), examinando la evidencia hoy dispersa sobre el vínculo entre liderazgo en el gobierno universitario y la capacidad de los docentes de innovar para mejorar la calidad de su enseñanza. Con este objetivo, el artículo se divide en cuatro apartados. En primer lugar, examinamos la relevancia de los diferentes estilos de liderazgo en las universidades, identificando las principales conclusiones de la literatura especializada sobre este tema. A continuación, exploramos la evidencia disponible sobre la relación entre liderazgo en el gobierno universitario e innovaciones docentes, subrayando cómo los estilos de liderazgos transformacionales y distribuidos caracterizados en la sección anterior, se encuentran en principio mejor posicionados para promover la experimentación en la docencia e institucionalizar nuevas metodologías de enseñanza dentro de la institución. La tercera sección discute esta evidencia a la luz de los principales desafíos que deben enfrentar los sistemas universitarios latinoamericanos, elaborando al final un modelo para comprender el vínculo entre liderazgo en el gobierno universitario e innovaciones docentes; útil tanto para la investigación como para los responsables de la gestión de estas instituciones. El ensayo termina con un breve resumen, líneas de investigación y una serie de sugerencias para mejorar la calidad de la enseñanza.

\section{Liderazgo en el gobierno universitario e innovaciones docentes}

En términos generales, el liderazgo es considerado como la capacidad de influir en otros y orientar los esfuerzos de los miembros de una organización para cumplir los objetivos de una institución específica (Erkutlu y Chafra, 2018). Hoy en día se reconoce en este sentido el potencial de los liderazgos para incidir en el logro de la eficacia social y operativa, y en definitiva en el éxito institucional (Croucher y Lacy, 2020). Bajo esta premisa es que el liderazgo es considerado como uno de los fenómenos organizacionales de mayor relevancia e interés en el campo de la educación superior (Pedraja-Rejas, Vega y Riquelme, 2018). Ahora bien, la literatura reconoce la existencia de diferentes estilos, aunque los que muestran mayor repercusión en esta área son: el liderazgo transformacional (Balwant, 2016; Brown, Brown y Nandedkar, 2019) y el liderazgo distribuido (Youngs, 2017; Vuori, 2019). ${ }^{2}$

\footnotetext{
${ }^{1}$ Reconocemos la existencia de un amplio espectro de teorías en torno a los liderazgos educativos en general. Sin embargo, considerando el objetivo de este estudio, la diferenciación entre liderazgos transformacionales y distribuidos resulta fundamental considerando su importancia en el campo de la educación superior.
} 
El estilo de liderazgo transformacional se caracteriza por una serie de rasgos distintivos. Primero, el líder transformacional genera una influencia idealizada basada principalmente en su carisma, lo que se traduce en respeto, admiración y confianza por parte de los seguidores, quienes tienden a imitar el actuar percibido en el líder (Udin et al., 2019; Gaus et al., 2020). Segundo, el líder transformacional logra alto niveles de motivación en sus equipos, creando un clima de compromiso en aras de alcanzar un futuro compartido mediante el esfuerzo conjunto (Cetin y Kinik, 2015; Ibrahim y Nat, 2019). Tercero, el líder transformacional es capaz de estimular intelectualmente a los miembros de su equipo, fomentando la creatividad e innovación (Al-Husseini y Elbeltagi, 2018; Al-Husseini, Elbeltagi y Moizer, 2019; Asiedu et al., 2020). Finalmente, el líder transformacional actúa considerando a cada miembro del equipo, en forma individual, apoyándolos y orientándolos de acuerdo con sus necesidades y capacidades de contribuir al proyecto general (Balwant, 2016; Majeed et al., 2017; Al-Amri et al., 2018). Por consiguiente, como lo sostienen desde hace décadas Bass y Avolio (1993) y Bass (1997), para las organizaciones en general, el liderazgo transformacional asume que la estructura de roles y su adecuado uso resultan esenciales para las acciones y decisiones institucionales.

A su turno, el liderazgo distribuido se singulariza por una serie de atributos. En primer lugar, el liderazgo distribuido genera cambios en la distribución del poder, control y la autoridad (Gosling, Bolden y Petrov, 2009; Vuori, 2019; Jones y Harvey, 2017). Segundo, el liderazgo distribuido se entiende como una práctica cotidiana más que como una función predefinida formalmente y que siga la estructura administrativa de la organización (Shankar y Sahin, 2017; Youngs, 2017; Walker, 2019). En tercer lugar, el énfasis del quehacer en el caso de este tipo de liderazgo está en las interacciones de las personas más que en sus acciones individuales (Beckman, 2017; Joslyn, 2018). Por último, el liderazgo distribuido no se restringe a lo formal, sino que reconoce que la informalidad es un elemento vital de las interacciones (Woods, 2016; Sewerin y Holmberg, 2017; Harvey y Jones, 2020). En consecuencia, como lo sostienen Harris (2007) y Harris y De Flaminis (2016), el liderazgo distribuido implica una comprensión de las organizaciones fundada principalmente en una perspectiva micropolítica, sin una asociación marcada con la estructura de roles.

Ambas formas de liderazgo, debido a su potencial por incidir en aspectos organizacionales claves, ha sido estudiado desde diversas posturas, perspectivas, metodologías y campos sociales (Navarro-Corona, 2016). En el ámbito de la educación superior en particular, el liderazgo ha tomado un lugar importante en la lente de los investigadores, tanto a nivel del gobierno como de los académicos, ya que impera hoy en día la necesidad de ejercerlo de manera sistemática, debido al potencial que éste tiene de promover la innovación organizativa, motivar a los seguidores, influir en los procesos formativos y en los resultados académicos, e incidir en la efectividad institucional (Ordorika, 1995; Ordorika, Martínez y Ramírez, 2011; Ganga et al., 2018; Pedraja-Rejas et al., 2019).

En concreto, los estudios de liderazgo en el gobierno universitario en este campo se han desarrollado por varias décadas (Middlehurst y Elton, 1992; Newton, 2002; Blaschke, Frost y Hattke, 2014). En particular, el impacto de dichos liderazgos en las universidades muestra relevancia en varias dimensiones, entre las cuales es posible destacar las siguientes: primera, hay evidencia de una relación entre liderazgo y las innovaciones en educación superior (Elrehail et al., 2018; Al-Husseini, Elbeltagi y Moizer, 2019; Asiedu et al., 2020); segunda, los estudios empíricos revelan que los estilos de liderazgo en las instituciones universitarias influyen en la cultura organizativa (Cruz y LaNise, 2017; Al-Kurdi, El-Haddadeh y Eldabi, 2018; Akanji et al., 2019); tercera, existe una relación explícita entre estilos de liderazgo y calidad de las instituciones (Trivellas y Dargenidou, 2009; 
Garwe, 2014; Rodríguez-Ponce, Pedraja-Rejas y Ganga-Contreras, 2019), y cuarta, los antecedentes empíricos indican también una relación de complementariedad entre el liderazgo y la eficacia en la educación superior (Siddique et al., 2011).

A pesar de esto, la mayoría de los estudios sobre liderazgo en el gobierno universitario y sus impactos en términos de innovaciones docentes se concentran en tres países: Estados Unidos, Reino Unido y Australia; por lo cual la investigación sobre esta materia es aún escasa, existiendo un amplio campo para avanzar en otras latitudes del mundo (Esen, Bellibas y Gumus, 2020). En este sentido, en términos generales, el impacto del liderazgo en la calidad de los procesos formativos en las instituciones de educación superior parece no haber sido abordado en detalle todavía. A pesar de su importancia, los estudios disponibles sobre este tema son todavía bastante limitados, estando la investigación en liderazgo en el gobierno universitario desacoplada del área de estudios de los procesos de enseñanza y aprendizaje en la educación superior, lo que resulta por lo tanto en pocos estudios sobre el vínculo entre dicho liderazgo e innovaciones docentes (Mumford et al., 2002; Robinson, Lloyd y Rowe, 2008; Gunn y Fisk, 2013). En la próxima sección revisamos los hallazgos de las principales investigaciones en este campo para luego proponer un marco de análisis para su comprensión específicamente en las universidades latinoamericanas.

\section{Liderazgo e innovaciones docentes: una revisión del estado del arte}

Como indica Quinlan (2014) a partir de una revisión de la literatura, el campo de investigación en liderazgo en el gobierno universitario, enseñanza y aprendizaje no recibe suficiente atención. Por una parte, existe una amplia discusión conceptual sobre los diferentes estilos de liderazgo en las instituciones de educación superior, frecuentemente vinculada a una crítica de los modelos jerárquicos o gerencialistas (Bolden et al.,
2008; Scott et al., 2008; Middlehurst, 2008) y, por otra, un número considerable de estudios centrados en el análisis de la agencia individual, como rectores de las universidades o las autoridades de las facultades, sin prestar mayor atención al análisis del contexto organizacional que otorga sentido y determina el impacto de las formas particulares de sus liderazgos (Spillane, Halverson y Diamond, 2004).

Este déficit resulta especialmente significativo cuando se considera que uno de los propósitos centrales del liderazgo en el gobierno universitario es mejorar la calidad de la enseñanza (Eison, 2002; Martin et al., 2003). En este sentido, el liderazgo de los directivos de las universidades, expresado, como proponen Álvarez Botello, Torres Velázquez y Chaparro Salinas (2015), tanto en términos de sus liderazgos institucionales, directivos y pedagógicos, permite promover el involucramiento de los miembros de la universidad en el objetivo de ayudar a mejorar la calidad del aprendizaje de los estudiantes.

Como punto de partida de nuestra revisión, entonces, es necesario apuntar que la innovación en la docencia no se explica por la existencia de individuos excepcionales, trabajando dentro de un paradigma de gestión tradicional, sino que resulta de prácticas a nivel de gobierno universitario que hacen posible liberal el potencial creativo de individuos ordinarios (Getz y Robinson, 2003). Tres aspectos resultan esenciales a este respecto según Marshall (2010). Primero, establecer una cultura en los distintos niveles de la institución que valore la docencia y posea sistemas de incentivos y recompensas adecuados para promover su calidad. Segundo, facilitar el acceso a sistemas para desarrollo profesional de los docentes y adquirir nuevas competencias y habilidades. Finalmente, fortalecer redes de comunicación entre estudiantes, docentes y directivos que incentiven el intercambio de ideas y trabajo en equipo.

La literatura especializada coincide en general en este diagnóstico. Como concluyen Barnard y Van der Merwe (2016), para que el liderazgo del gobierno 
universitario afecte positivamente la capacidad de los docentes de innovar para mejorar la calidad de la enseñanza se requiere de un trabajo administrativo que involucre actividades de planificación, discusiones, revisiones constantes y, en general, la promoción de procesos internos de reflexión organizacional. En una dirección similar, McInnis, Ramsden y Maconachie (2012) indican que, a nivel de gestión estratégica central, es necesario que los líderes posean una visión clara de la docencia, sean capaces de identificar y desarrollar liderazgos académicos e involucrar a los estudiantes en el proceso de toma de decisiones. Quinlan (2014) ofrece en este sentido, a nuestro juicio, una buena síntesis de estas ideas, indicando que para que el liderazgo del gobierno universitario resulte en una mejor calidad de la docencia se requiere crear condiciones organizacionales adecuadas para el aprendizaje, una voluntad para implementar nuevos valores institucionales centrados en el estudiante y una disposición a aprender de las experiencias propias y ajenas, transversal en la institución, para compartir buenas prácticas e identificar innovaciones positivas.

Por otro lado, en relación con el tema del gobierno universitario y su influencia en la generación de innovaciones positivas en la docencia existen, de acuerdo con la literatura, una serie de obstáculos a considerar. En primer lugar, la existencia de contradicciones en los sistemas de incentivos de la institución, especialmente respecto de la investigación y la docencia, es un aspecto especialmente relevante, en tanto puede redundar en una subvaloración de la segunda frente a la primera y la consecuente falta de interés de los académicos en otorgar una docencia de calidad por considerar esta tarea como una carga a evitar que no contribuye directamente al positivo desarrollo de su carrera profesional (Ramsden, 1998).

A continuación, en aquellas instituciones en que, desde el gobierno universitario, se impone una lógica competitiva, existe la tendencia entre los docentes a trabajar de manera independiente entre sí. Esto resulta en una falta de intercambio de experiencias docentes y una reducción de la posibilidad de aprender de las metodologías empleadas por otros profesores (Klein y Falk-Krzensinski, 2017).

Tercero, la burocratización de los procesos de enseñanza, impulsada desde el gobierno central, suele elevar el tiempo que los docentes deben dedicar a reportar sus actividades, restringiendo por tanto su dedicación a la experimentación con nuevos métodos de enseñanza. Cuando esto ocurre, como han apuntado Aitken y O’Carroll (2020) y Rodríguez Pulido, Artiles Rodríguez y Aguiar Perera (2015) para los casos de las universidades británicas y españolas, respectivamente, se genera una disociación entre los lineamientos de los estamentos directivos y los académicos que finaliza deslegitimando los liderazgos y planes de los primeros.

Finalmente, la existencia de liderazgos insuficientemente cualificados en el gobierno universitario parece también influir negativamente en la calidad de la docencia universitaria (Igwe et al., 2019). Esta falta de competencias resulta especialmente relevante cuando va asociada con una estructura organizacional que no otorga apoyo administrativo para la innovación y que carece de los recursos suficientes para poder impulsar estas actividades (Marshall, 2010).

Para superar estos obstáculos, la experiencia comparada subraya la centralidad de dos tipos de liderazgo en el gobierno universitario. Por una parte, existen una serie de estudios que enfatizan la relevancia de los liderazgos transformacionales en fomentar el desarrollo de espacios organizacionales que mejoren la calidad de la enseñanza. A diferencia de los liderazgos transaccionales, en que las personas desempeñan sus tareas en intercambio de una retribución, sea esta económica o simbólica, los liderazgos transformacionales se basan en el carisma del líder y su habilidad para motivar a que las personas realicen sus mayores esfuerzos por los sentimientos de confianza, lealtad e inspiración que éste puede generar (Bass et al., 2003; Le y Lei, 2019; Zuraik y Kelly, 2019). 
Los líderes transformacionales son en este sentido capaces de articular su visión de manera tal que resulta motivante para las personas que forman parte de la institución, impulsando una nueva manera de comprender el proceso de enseñanza y aprendizaje dentro de la institución (Erkutlu, 2008). Como arguyen Al-Husseini y Elbeltagi (2016), los liderazgos directivos transformacionales en el gobierno universitario son capaces de estimular el pensamiento innovador de sus comunidades, esto es, analizar problemas de manera diferente, reconocer las contribuciones de distintas personas y crear espacios para innovar en el diseño de cursos, proyectos de investigación, currículos y herramientas tecnológicas.

A tal efecto, como sostienen Bass y Riggio (2006), los líderes con foco en la transformación permiten reinterpretar los problemas, creando el ambiente para buscar nuevas soluciones. La generalización de dicha competencia resulta clave, en tanto que permite avanzar desde los órganos directivos centrales hacia una cultura de la innovación en las universidades, promoviendo la experimentación metodológica y el aprendizaje organizacional de buenas prácticas pedagógicas (Gumusluoglu y Ilsev, 2009; Eisenbeig y Boerner, 2013). Esto se traduce luego en políticas de desarrollo docente de las unidades académicas, y en el diseño de planes para promover e impulsar las iniciativas de enseñanza y aprendizaje adecuadas para las necesidades de los estudiantes (Kouzes y Posner, 2019; Scott y Guthrie, 2003).

Diversos estudios empíricos apuntan en esta dirección. Asiedu et al. (2020), por ejemplo, examinan el vínculo entre liderazgo transformacional, capacidades de administración de conocimiento, aprendizaje organizacional e innovación en las instituciones de educación superior de Ghana. Según su análisis, el liderazgo transformacional promueve la creatividad y desarrolla las capacidades cognitivas, la innovación y la colaboración entre académicos. Por su parte, en un estudio del sistema universitario hindú, se concluye que los liderazgos transformacionales resultan en una mejor comunicación, expectativas claras entre directivos y docentes y el reconocimiento de los aportes particulares de cada uno (Jyoti y Bhau, 2016).

A su vez, en relación con los comportamientos de los docentes universitarios bajo este tipo de líderes en el gobierno central, Koeslag-Kreunen et al. (2021), examinando una universidad holandesa, subrayan que este estilo de liderazgo es capaz de incentivar de hecho el trabajo en equipo en la institución. A una conclusión similar llegan Lodders y Meijirs (2017), igualmente respecto de las universidades holandesas, señalando que los liderazgos transformacionales favorecen el aprendizaje colectivo y aumentan la capacidad de innovación tanto a nivel de la docencia como de la investigación.

En el contexto latinoamericano existe evidencia de un impacto similar. Según un estudio de Jaime Guadros, Cáceres Reche e Hinojo Lucena (2018) en una universidad colombiana, la existencia de liderazgos directivos transformacionales resulta en una mayor satisfacción de los profesores, un mejor manejo de conflictos y resolución de problemas y una mejor docencia. Por su parte, Durey et al. (2017), evaluando un programa formativo intercultural con liderazgo transformacional, indican que éste facilita reconocer las diferencias en el proceso de enseñanza.

A mayor abundamiento es posible identificar además análisis que sugieren que los impactos de los liderazgos transformacionales en la educación superior no se restringen sólo a la enseñanza de los docentes, sino que se extienden al aprendizaje de los estudiantes. En este sentido, el liderazgo transformacional en el gobierno de la universidad influye en la satisfacción de los estudiantes, como muestra el estudio de Bogler, Caspi y Roccas (2013) de una universidad en Israel. De la misma manera, en relación con instituciones de Hong Kong, Pounder, Stoffel y Choi (2018) concluyen que este estilo de liderazgo mejora la calidad de la docencia, elevando la empleabilidad de los egresados. Finalmente, la investigación de AlHusseini y Elbeltagi (2016) en el sistema universitario 
de Irak sugiere que los liderazgos transformacionales contribuyen a crear un ambiente de enseñanza enfocado en innovación que facilita el aprendizaje por parte de los estudiantes.

Otra línea de estudios sobre el liderazgo destaca la centralidad de los liderazgos distribuidos en promover la innovación en la docencia universitaria. Como exploramos anteriormente, el liderazgo distribuido corresponde a un liderazgo emergente y distribuido a lo largo de una institución, donde la confianza organizacional y el conocimiento del contexto resultan más fundamentales que la posición, la cultura es más determinante que el control jerárquico y, en lugar de la competencia, se valora la colaboración desde las propias especialidades y experticias y la construcción de una identidad común (Bennett et al., 2003; Jones et al., 2012). Específicamente, en relación con el impacto de este tipo de liderazgo en el gobierno universitario en la enseñanza, la literatura coincide en señalar que puede contribuir a reducir la distancia entre personal de gestión y docencia (Jones et al., 2012; Harkin y Healy, 2013), impulsar el cambio curricular ante desafíos (Keppell et al., 2010) y aumentar la innovación pedagógica (Creanor, 2014).

La principal causa de estos efectos, según apuntan estos estudios, es la mayor participación que va asociada a este tipo de liderazgo. En efecto, Pepper y Roberts (2016) concluyen que la existencia de un liderazgo distribuido en las universidades facilita el reconocimiento de líderes en posiciones formales e informales, recogiendo la experiencia de directivos y académicos en distintos niveles de decisión y facilitando el aprendizaje interno de buenas prácticas. De manera similar, esta opción permitiría involucrar a los docentes en el proceso de toma de decisiones, aumentando su compromiso e identificación con los fines de la institución y, por lo tanto, su motivación para mejorar la calidad de su enseñanza e innovar en sus prácticas pedagógicas (Moolenaar et al., 2010).

La literatura comparada recoge diferentes experiencias en este sentido, especialmente en el contexto australiano. Jones et al. (2017), analizando una universidad de este país, arguyen que el liderazgo distribuido permite de hecho mejorar las capacidades de liderazgo de la institución, aumentar el involucramiento de los académicos, sistematizar las prácticas colaborativas y facilitar el cambio sistémico tanto en los repertorios de enseñanza como de evaluación. A su vez, Bianchini, Maxwell y Dovey (2014) subrayan que distribuir el proceso de toma de decisiones a lo largo de una universidad australiana, incorporando liderazgos de académicos y partes interesadas, contribuye a elevar la capacidad de autorregulación de la institución y a adaptar las herramientas relacionadas con la planificación de la docencia a las demandas desde los estudiantes.

En otras partes del mundo se llega a conclusiones semejantes. Bolander Laksov y Tomson (2017) sostienen que la participación impulsada desde el gobierno universitario por los liderazgos distribuidos resulta clave para mejorar la calidad de la enseñanza de la educación médica en Suecia. En el mismo sentido, en relación con la educación superior STEM (Science, Technology, Engineering and Mathematics) en Estados Unidos, Borrego y Henderson (2014) destacan cómo este estilo de liderazgo a nivel central mejora el intercambio de información y promueve el debate sobre los objetivos específicos de la institución, aumentando su legitimidad. Por su parte, en su análisis de una universidad sudafricana, Cronje y Bitzer (2019) destacan que el liderazgo distribuido contribuye a empoderar a los docentes en sus decisiones, dándoles más espacio para la innovación y la experimentación. Finalmente, en una universidad mexicana, Velázquez Hernández y Hernández Romero (2019) enfatizan cómo los procesos de democratización del gobierno institucional asociados al aprovechamiento de liderazgos distribuidos incentivan una mayor responsabilización de los profesores sobre la calidad de su docencia, facilitando el aprendizaje organizacional.

Como puede derivarse a partir de esta revisión, el liderazgo en el gobierno universitario - tanto en su 
versión transformacional como distribuida - resulta esencial para la innovación orientada a mejorar la calidad de la docencia. Sin embargo, en este sentido, es preciso recordar que no existe una solución del tipo one-size-fits-all sino que la pertinencia de cada tipo de liderazgo en la docencia se determina según las características específicas del contexto, en particular, la cultura de las unidades académicas locales y la estructura de la universidad y los lineamientos a nivel de economía política de cada sistema nacional de educación superior (Brunner et al., 2021).

$\mathrm{Al}$ respecto, el estudio de Gibbs et al. (2008) de 19 departamentos exitosos en universidades de investigación, concluye que la calidad de la enseñanza puede ser en la práctica alcanzada con distintos tipos de liderazgo a nivel de gobierno central. Lo que funciona en una institución puede por tanto no hacerlo en otra, en tanto el liderazgo adecuado, así como su pertinencia como propulsor de una docencia de calidad con foco en la innovación, depende así del contexto (Bleiklie et al., 2017).

Los departamentos son usualmente descritos aquí como las principales unidades académicas de liderazgo a impulsar desde el nivel central al tomar en cuenta decisiones relacionadas con la docencia, en tanto en esta formación se combinan las directrices generales de la institución, junto con los lineamientos característicos de la política pública, con las prioridades derivadas de la cultura local de directivos, académicos y estudiantes (Martin et al., 2003). Los liderazgos locales resultan entonces fundamentales para reformar las culturas de aprendizaje.

Es preciso notar que los liderazgos locales - y esta es la principal ventaja de los departamentos - consideran líderes formales e informales. Mientras los líderes formales, como rectores y vicerrectores, junto con directores de departamento y jefes de carrera, tienen un rol central en la asignación de recursos y en la implementación de sistemas de incentivos y de reconocimiento de innovaciones docentes, los líderes informales, justamente por su cercanía a los valores locales de las respectivas unidades, tienen la ventaja de poder asegurar la legitimidad de las iniciativas de reforma educativa e impulsar su eficacia más allá del plano discursivo (Ramsden et al., 2007; Bates y Sangra, 2011). Como apuntan Ng'ambi y Bozalek (2013) y Thornton et al. (2018) sobre universidades de Sudáfrica y Nueva Zelanda, respectivamente, la combinación de ambos liderazgos puede acelerar la difusión de prácticas transformacionales, mediante distintas formas de trabajo en equipo, ayudando a que sean reconocidas en sus respectivas instituciones y puedan estabilizar innovaciones a nivel docente.

\section{El desafío de liderazgos para una docencia de calidad en América Latina}

Si bien no se ha analizado sistemáticamente el vínculo entre liderazgos en el gobierno universitario e innovaciones docentes en América Latina, este tema resulta especialmente importante en el contexto latinoamericano. Los sistemas de la región se han enfrentado a los tres desarrollos descritos en la introducción -masificación, relevancia de competencia en la economía de la información y el conocimiento, y aumento de la importancia de recursos por concepto de matrícula - en el contexto de un modelo de desarrollo económico marcadamente neoliberal (Bonal, 2011), lo que hizo que dichas transformaciones, a diferencia de lo ocurrido en otras regiones, se expresasen en un marco en que la matrícula fue absorbida principalmente por instituciones privadas (Brunner y Miranda, 2016); la formación universitaria pasó a ser concebida como un bien privado, capital humano para aumentar la productividad de las personas (Tünnerman, 2003) y se impulsó la competencia entre instituciones por recursos fiscales y privados (García Guadilla, 2007), incrementándose de manera notable los ingresos provenientes de las fuentes privadas y alterando los fines tradicionales de la docencia (Brunner et al., 2019).

Como resultado, las universidades de la región se han visto forzadas a reformar sus gobiernos internos, 
prestando mayor atención a la cuestión de si sus estilos de liderazgos en este nivel son efectivamente adecuados para enfrentar estos nuevos desafíos. Estas reflexiones han generado un apasionado debate político sobre los modelos de liderazgo más adecuados para las instituciones de la región, arguyéndose así sobre las ventajas y desventajas de un "sistema tradicional", centrado en el liderazgo de los académicos y estudiantes, versus un "sistema emprendedor", con una mayor centralidad en los procesos de decisión de los estamentos directivos de la universidad (Tünnermann, 2003; Pineda, 2015).

En este contexto, la cuestión del liderazgo en el gobierno central de las universidades latinoamericanas ha recibido una notable atención, a diferencia de lo que ocurre con el vínculo entre estilos de liderazgo e innovaciones docentes (Pérez, Guarín y Romo, 2015; Ganga et al., 2018). Sin embargo, si recogemos las principales conclusiones de la sección anterior, es posible identificar una serie de dimensiones que resultan relevantes para avanzar en la investigación sobre este tema, de manera tal que permitan además promover procesos de reflexión internos en las instituciones de educación sobre sus retos al respecto.

En este sentido, en primer lugar, como apunta la literatura, no existe un único tipo de liderazgo efectivo para asegurar la calidad de la docencia. Como vimos anteriormente, dos estilos de liderazgo a nivel de gobierno universitario parecen ser especialmente relevantes en este escenario: el liderazgo transformacional, centrado en el carisma del directivo y la lealtad que éste puede generar entre sus académicos, y el liderazgo distribuido, enfocado en promover la participación, experticia y autonomía de los distintos actores de la institución. Para ambos casos existe evidencia de experiencias exitosas. Por lo tanto, el debate no debe enmarcarse en cuál liderazgo permite un mayor número de innovaciones, sino en cambio qué estilo de liderazgo es adecuado considerando, por una parte, la trayectoria histórica de la institución, junto con sus tradiciones y, por otra, sus desafios a mediano y largo plazo en el área de la enseñanza.

En segundo lugar, y relacionado con lo anterior, la eficacia de dichos liderazgos se juega en distintos niveles, pero el más importante de ellos es la unidad local, esto es, el departamento. En efecto, éste es el espacio de combinación de las directrices emanadas de los liderazgos centrales de la política pública (ministros, subsecretarios, etcétera) y de la institución (rectores y vicerrectores) con los liderazgos directivos formales e informales de la unidad académica que, por su cercanía al día a día de académicos y estudiantes, poseen un conocimiento mucho más estrecho de las dinámicas de enseñanza y aprendizaje. El resultado son innovaciones docentes emergentes, no completamente determinadas de manera top-down ni bottom-up, sino que son resultado de su modo peculiar de interacción en cada unidad.

Tercero, estas unidades se componen de una estructura organizacional y una cultura particular que no necesariamente se encuentran alineadas entre sí, restringiendo en este caso la posibilidad de vincular las decisiones de los líderes con la promoción de innovaciones docentes. A modo de ejemplo, la cultura de un departamento, formalizada a nivel de sus directrices directivas y expresada en los presupuestos no discutidos por parte de sus miembros, puede subrayar el foco en la innovación pedagógica y el desarrollo de actividades centradas en el estudiante, pero, al mismo tiempo, a nivel de la organización, asignar mayores recompensas, económicas y simbólicas, a la investigación en desmedro de la docencia. El liderazgo en el gobierno universitario orientado a la docencia de calidad, ya de por sí improbable, se ve seriamente afectado en estos casos, esto es, cuando no es capaz de impulsar efectivamente transformaciones a nivel de los departamentos de las instituciones.

Por último, es preciso reconocer la influencia de la universidad y el sistema nacional de educación superior en la relación entre liderazgo en el gobierno universitario e innovaciones docentes. Ciertamente, este vínculo no es tan directo como en el caso de los 
departamentos, pero de todas maneras este plano, asociado usualmente a decretos y reglamentos para el modo de toma de decisiones en las instituciones estatales en particular, otorgan un marco general de definiciones sobre el liderazgo que es traducido efectivamente en el nivel local. En este sentido, la orientación del gobierno universitario, así como su grado de descentralización, pueden o no dar espacio para la emergencia de un liderazgo transformacional, distribuido o de otro tipo, con mayor o menor resistencia según los lineamientos definidos en este nivel. En una dirección similar, el sistema nacional de educación superior, con sus decisiones correspondientes en términos de financiamiento y regulación, orienta el desarrollo de ciertos tipos de liderazgo en las instituciones, como ocurre hoy con la tendencia a liderazgos de tipo emprendedor dentro de las universidades. La figura 1 sintetiza estas ideas.

Figura 1. Modelo de innovación docente desde la perspectiva del liderazgo en el gobierno de la universidad

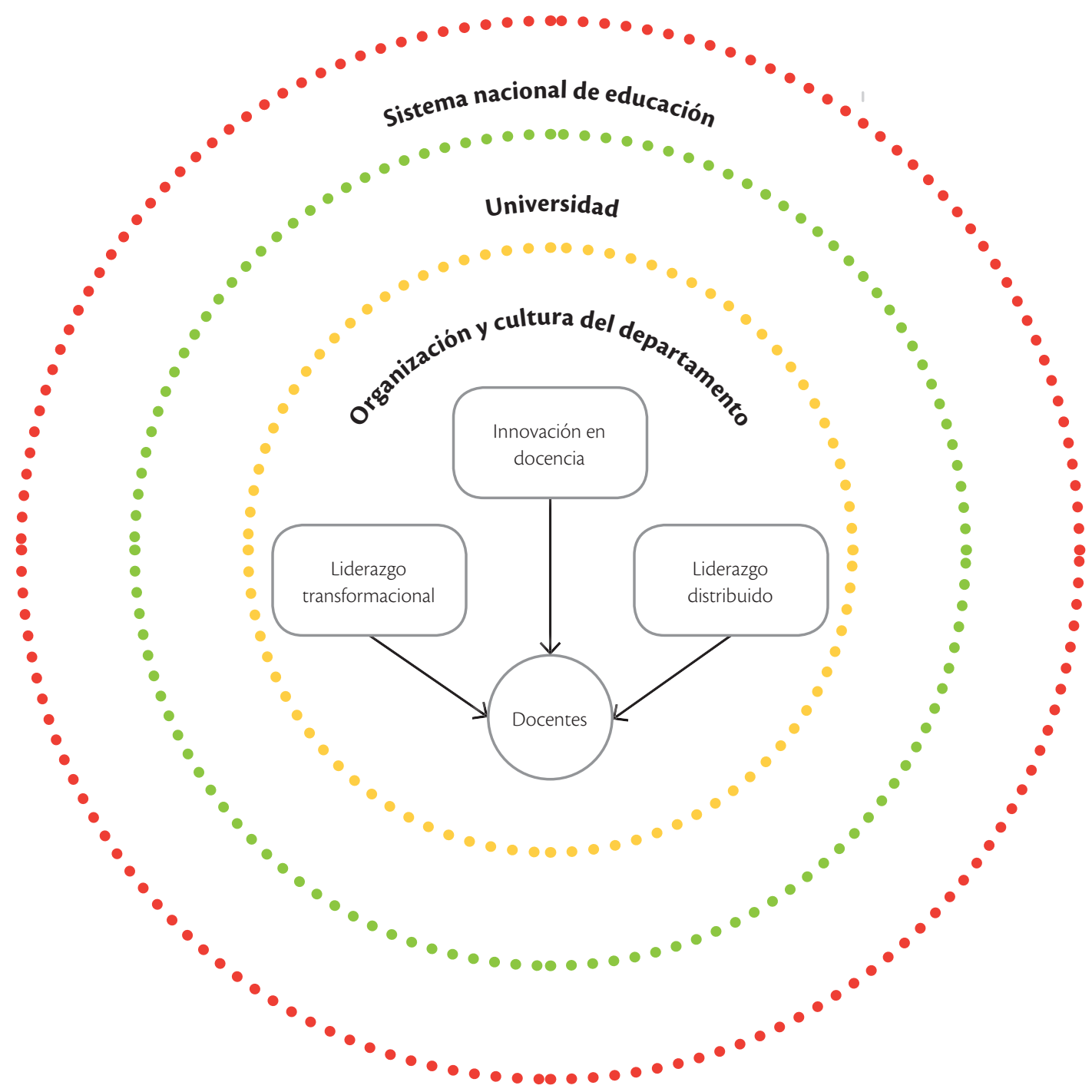




\section{Conclusiones}

En este ensayo hemos examinado la evidencia disponible sobre el impacto del liderazgo de los directivos universitarios en la implementación de innovaciones en el proceso de enseñanza de los docentes. Con base en lo anterior propusimos un modelo para comprender el vínculo entre liderazgo en el gobierno universitario e innovaciones docentes con la expectativa de que pueda ser empleado, por un lado, como un medio para sintetizar la literatura científica sobre este tema y avanzar en esta línea de investigación y, por otra, para facilitar los procesos de reflexión organizacional en las universidades latinoamericanas y así aumentar sus capacidades de respuesta frente a entornos crecientemente complejos con base en la evidencia disponible.

En relación con la investigación sobre liderazgo en el gobierno universitario e innovación docente en las universidades, dos líneas futuras de análisis nos parecen particularmente interesantes. En primer lugar, es necesario examinar las diferencias en los estilos de liderazgo en este nivel entre distintas instituciones de educación superior y sus efectos en términos de facilitar la emergencia de innovaciones en el proceso de enseñanza-aprendizaje. En este punto la literatura especializada recoge la existencia de profundas diferencias en los estilos de conducción a nivel central de las instituciones de educación superior estatales y privadas, con las primeras incorporando generalmente un mayor número de mecanismos participativos y un proceso más horizontal de toma de decisiones que sus contrapartes (Nizami y Rashid, 2018; Alarcón, 2020). En este sentido, si bien existe un número importante de estudios que compara los diferentes estilos de liderazgo directivo en el gobierno de las universidades, la manera en que dichos estilos influyen en términos prácticas en las actividades docentes parece ser todavía una incógnita. Según lo aquí revisado, los liderazgos transformacionales y distribuidos en el gobierno universitario se encuentran en una posición privilegiada para crear un ambiente de experimentación y valoración de las innovaciones en los departamentos, si bien es necesario desarrollar más estudios empíricos que reconozcan la influencia de las trayectorias de las instituciones, la economía política y el modo general de gobernanza, con especial foco en la región latinoamericana.

Por otro lado, resulta conveniente examinar cómo los estilos de liderazgo de las universidades a nivel central influyen efectivamente en la capacidad de los docentes de innovar en sus procesos de enseñanza. Que existe un vínculo es uno de los principales hallazgos de los estudios revisados, con mucha menos información disponible sobre cuáles son los factores organizacionales que promueven la generalización de un enfoque innovador entre directivos y académicos con efectos reales en su docencia. En este sentido, no resulta suficiente sólo la presencia de liderazgos a nivel central, sino que éstos deben ser capaces de influir positivamente en las prácticas de los docentes (a modo de ejemplo, véase Balwant, 2017). La idea de cultura universitaria - que hemos desarrollado en otras investigaciones (Pedraja-Rejas, Rodríguez-Ponce y Labraña, 2021) - nos parece especialmente interesante en este contexto, en tanto permite presuponer a nivel social la existencia de orientaciones normativas suficientemente reconocidas en la institución y que hacen posible, gracias a lo anterior, dirigir la conducta de sus miembros hacia objetivos comunes. En el caso que nos convoca - referido al vínculo entre liderazgo en el gobierno central e innovación docente en las universidades-, la presencia de una cultura orientada al cambio y la experimentación facilita que las transformaciones impulsadas a nivel directivo sean incorporadas como guías de acción en las labores de los docentes y no se queden simplemente como una mera decisión administrativa. Para confirmar o rechazar dicha suposición se requiere sin embargo investigación empírica sobre los estilos de liderazgo y su impacto en la docencia desde una perspectiva organizacional y culturalista. 
Como señalamos en un comienzo, el modelo propuesto para entender la relación entre el liderazgo de los directivos de nivel central y las innovaciones en el proceso de enseñanza no tiene sólo fines académicos, sino que aspira a ser empleado como un elemento para facilitar la autoobservación de las universidades sobre sus procesos. En este aspecto queremos finalizar el presente ensayo con dos ideas. En primer lugar, en general, en el gobierno de las instituciones de la región predomina un modelo colegial con liderazgos poco claros, distribuidos a nivel de las facultades. Mucho se ha discutido si este modelo es adecuado para enfrentar los desafios del futuro y si es necesario avanzar en un sentido más emprendedor (Fossatti, Ganga y Jung, 2017). A nuestro juicio, más importante para las universidades que decidir esta cuestión conceptualmente, es examinar cuáles son los efectos de estos estilos de liderazgo y si cumplen con el objetivo de asegurar la calidad de la enseñanza. El marco aquí propuesto puede servir para examinar estos fines en mayor detalle.

Segundo, es preciso incorporar al nivel de los sistemas de aseguramiento de la calidad una dimensión relacionada con el liderazgo de las universidades. Como apuntamos en este trabajo, el liderazgo de los directivos tiene importantes efectos en la calidad de la docencia. En este sentido, si se toma en cuenta que la mayoría de las instituciones de educación superior de la región latinoamericana desempeñan tareas principalmente docentes (Brunner y Miranda, 2016), el tema de la evaluación de las variables que influyen en su calidad se convierte en central cuidando que éstas puedan extender sus efectos a los departamentos. Sin embargo, si se examinan los sistemas de la región, la cuestión del liderazgo en el gobierno universitario y sus consecuencias sobre la innovación docente parece encontrarse largamente ausente, especialmente si se compara con la situación en otros sistemas nacionales (Alzafari y Kratzer, 2019). La reforma de los métodos de evaluación de la calidad de las instituciones en esta dirección es recomendable en este punto.

Como examinamos en este ensayo, el liderazgo directivo a nivel central es una variable cuya importancia en la calidad de la docencia universitaria debe ser adecuadamente reconocida. Esperamos que el presente ensayo, junto con el modelo de análisis propuesto, pueda servir a su discusión tanto en la investigación académica como en el diseño de políticas públicas. 


\section{Agradecimientos}

Los autores agradecen a la Agencia Nacional de Investigación y Desarrollo, ANID por el patrocinio recibido a través del proyecto FONDECyT 1180484.

\section{Referencias}

Aitken, Gillian y Sinéad O'Carroll (2020), "Academic identity and crossing boundaries: the role of the Programme Director in postgraduate taught programmes", Higher Education Research \& Development, vol. 39, núm. 7, pp. 1410-1424.

Akanji, Babatunde, Chima Mordi, Afam Ituma, Toyin Ajibade Adisa y Hakeem Ajonbadi (2019), "The influence of organisational culture on leadership style in higher education institutions", Personnel Review, vol. 49, núm. 3, pp. 709-732.

Al-Amri, Ammar Y., Roshidi Hassan, Yassien Masoud y Osama Isaac (2018), "The impact of transformational management on organizational innovation in higher education: the case of growing countries", International Journal of Management and Human Science, vol. 2, núm. 4, pp. 25-37.

Alarcón, Mario (2020), El gobierno de la universidad desde la perspectiva de la teoría de agencia: abriendo la caja negra, Santiago, Chile, Políticas Comparadas de Educación, Facultad de Educación, Universidad Diego Portales/ Facultad de Humanidades, Universidad de Leiden, Holanda.

Al-Husseini, Sawasn e Ibrahim Elbeltagi (2018), "Evaluating the effect of transformational leadership on knowledge sharing using structural equation modelling: the case of Iraqi higher education", International fournal of Leadership in Education, vol. 21, núm. 4, pp. 506-517.

Al-Husseini, Sawasn e Ibrahim Elbeltagi (2016), "Transformational leadership and innovation: a comparison study between Iraq's public and private higher education", Studies in Higher Education, vol. 41, núm. 1, pp. 159-81.

Al-Husseini, Sawasn, Ibrahim Elbeltagi y Jonathan Moizer (2019), "Transformational leadership and innovation: the mediating role of knowledge sharing amongst higher education faculty", International Fournal of Leadership in Education, pp. 1-24.

Al-Kurdi, Osama, Ramzi El-Haddadeh y Tillal Eldabi (2018), "Knowledge sharing in higher education institutions: a systematic review", fournal of Enterprise Information Management, vol. 31, núm. 2, pp. 226-246.

Alonso-Sáez, Israel, Leire Darretxe y Nekane Beloki (2019), "Hacia una identidad y cultura académica colaborativa: los equipos docentes como innovación en los grados universitarios", Archivos Analíticos de Políticas Educativas, vol. 27, núm. 142, pp. 1-17.

Álvarez Botello, Julio, Alejandra M. Torres Velázquez y Eva M. Chaparro Salinas (2015), "Diagnóstico del liderazgo educativo en las instituciones de educación superior del Valle de Toluca", Revista de Investigación Educativa, vol. 34, núm. 1, pp. 51-68.

Alzafari, Khaled y Jan Kratzer (2019), "Challenges of implementing quality in European higher education: an expert perspective", Quality in Higher Education, vol. 25, núm. 3, pp. 261-88.

Asiedu, Mercy Asaa, Hod Anyigba, Kwame Simpe Ofori, George O. A. Ampong y John Agyekum Addae (2020), "Factors influencing innovation performance in higher education institutions", The Learning Organization, vol. 27, núm. 4, pp. 365-378.

Balwant, Paul (2017), "The dark side of teaching: destructive instructor leadership and its association with students' affect, behaviour, and cognition", International Fournal of Leadership in Education, vol. 20, núm. 5, pp. 577-604.

Balwant, Paul (2016), "Transformational instructorleadership in higher education teaching: a metaanalytic review and research agenda", fournal of Leadership Studies, vol. 9, núm. 4, pp. 20-42.

Barnard, Zenia y Derek Van der Merwe(2016), "Innovative management for organizational sustainability in higher education", International Fournal of Sustainability in Higher Education, vol. 17, núm. 2, pp. 208-227.

Bass, Bernard (1997), "Does the transactionaltransformational leadership paradigm transcend 
organizational and national boundaries?", American Psychologist, vol. 52, núm. 2, pp. 130-139.

Bass, Bernard M., Bruce J. Avolio, Dong I. Jung y Yair Berson (2003), "Predicting unit performance by assessing transformational and transactional leadership", fournal of Applied Psychology, vol. 88, núm. 2, pp. 207-218.

Bass, Bernard y Bruce Avolio (1993), "Transformational leadership: a response to critiques", en Martin M. Chemers y Roya Ayman (eds.), Leadership theory and research: perspectives and directions, San Diego, Academic Press, pp. 49-80.

Bass, Bernard y Ronald Riggio (2006), Transformation leadership, New Jersey, Lawrence Erlbaum Associates.

Bates, Tony y Albert Sangra (2011), Managing technology in higher education: strategies for transforming teaching and learning, California, Jossey-Bass.

Beckman, Elizabeth (2017), "Leadership through fellowship: distributed leadership in a professional recognition scheme for university educators", fournal of Higher Education Policy and Management, vol. 39, núm. 2, pp. 155-168.

Bennett, Nigel, Christine Wise, Philip Woods y Janet Harvey (2003), Distributed leadership: a review of literature, Notthingham, UK, National College for School Leadership.

Bianchini, Stefano, Tudor Maxwell y Ken Dovey (2014), "Rethinking leadership in the academy: an Australian case", Innovations in Education and Teaching International, vol. 51, núm. 5, pp. 556-567.

Blaschke, Steffen, Jetta Frost y Fabian Hattke (2014), "Towards a micro foundation of leadership, governance, and management in universities", Higher Education, vol. 68, pp. 711-732.

Bleiklie, Ivar, Nicoline Frølich, Rachel Sweetman y Mary Henkel (2017), "Academic institutions, ambiguity and learning outcomes as management tools", European Fournal of Education, vol. 52, núm. 1, pp. 68-79.

Bogler, Ronit, Avner Caspi y Sonia Roccas (2013), "Transformational and passive leadership", Educational
Management Administration \&̊ Leadership, vol. 41, núm. 3, pp. 372-392.

Bolander Laksov, Klara y Tanja Tomson (2017), "Becoming an educational leader - exploring leadership in medical education", International Journal of Leadership in Education, vol. 20, núm. 4, pp. 506-516.

Bolden, Richard, Georgy Petrov y Jonathan Gosling (2008), "Tensions in higher education leadership: towards a multi-level model of leadership practice", Higher Education Quarterly, vol. 62, núm. 4, pp. 358-376.

Bonal, Xavier (2011), "Plus ca change... the World Bank global education policy and the post-Washington consensus", International Studies in Sociology of Education, vol. 12, núm. 1, pp. 3-22.

Borrego, Maura y Charles Henderson (2014), "Increasing the use of evidence-based teaching in STEM higher education: a comparison of eight change strategies", Journal of Engineering Education, vol. 103, núm. 2, pp. 220-252.

Brown, Melissa, Roger S. Brown y Ankur Nandedkar (2019), "Transformational leadership theory and exploring the perceptions of diversity management in higher education", fournal of Higher Education Theory \& Practice, vol. 19, núm. 7, pp. 11-21.

Brunner, José J. y Daniel A. Miranda (2016), Educación superior en Iberoamérica: informe 2016, Santiago, Chile, Centro Interuniversitario de Desarrollo.

Brunner, José Joaquín, Julio Labraña, Emilio RodríguezPonce y Francisco Ganga (2021), "Variedades de capitalismo académico: un marco conceptual de análisis", Archivos Analíticos de Políticas Educativas, vol. 29, núm. 35, pp. 1-32.

Brunner, José Joaquín, Julio Labraña, Francisco Ganga y Emilio Rodríguez-Ponce (2019), "Idea moderna de universidad: de la torre de marfil al capitalismo académico", Educación XXI, vol. 22, núm. 2, pp. 1-22.

Cetin, Munevver Olcum y Sehkar Fayda Kinik (2015), "An analysis of academic leadership behavior from the perspective of transformational leadership", ProcediaSocial and Behavioral Sciences, vol. 207, pp. 519-527. 
Creanor, Linda (2014), "Raising the profile: an institutional case study of embedding scholarship and innovation through distributive leadership", Innovations in Education and Teaching International, vol. 51, núm. 6, pp. 573-583.

Cronje, Franci y Eli Bitzer (2019), "Continuous professional learning in private higher education: making a case for distributed leadership", South African Journal of Higher Education, vol. 33, núm. 2.

Croucher, Gwilym y William B. Lacy (2020), "Perspectives of Australian higher education leadership: convergent or divergent views and implications for the future?", Journal of Higher Education Policy and Management, vol. 42, núm. 4, pp. 516-529.

Cruz, Laura y Rosemond LaNise (2017), "Coaching academia: the integration of coaching, educational development, and the culture of higher education", fournal on Excellence in College Teaching, vol. 28, núm. 4, pp. 83-108.

Durey, Angela, Kate Taylor, Dawn Bessarab, Marion Kickett, Sue Jones, Julie Hoffman, Helen Flavell y Kim Scott (2017), "'Working together': an intercultural academic leadership programme to build health science educators' capacity to teach indigenous health and culture", The Australian Fournal of Indigenous Education, vol. 46, núm. 1, pp. 12-22.

Eisenbeig, Silke y Sabine Boerner (2013), "A doubleedged sword: transformational leadership and individual creativity", British fournal of Management, vol. 24, núm. 1, pp. 54-68.

Eison, James (2002), "Teaching strategies for the twentyfirst century", en Robert Diamond (ed.), Field Guide to Academic Leadership, San Francisco, Jossey-Bass, pp. 157-173.

Elrehail, Hamzah, Okechukwu Lawrence Emeagwali, Abdallah Alsaad y Amor Alzghoul (2018), “The impact of transformational and authentic leadership on innovation in higher education: the contingent role of knowledge sharing", Telematics and Informatics, vol. 35, núm. 1, pp. 55-67.

Erkutlu, Hakan (2008), "The impact of transformational leadership on organizational and leadership effectiveness: the Turkish case", Journal of Management
Development, vol. 27, núm. 7, pp. 708-26.

Erkutlu, Hakan y Jamel Chafra (2018), "Despotic leadership and organizational deviance", fournal of Strategy and Management, vol. 11, núm. 2, pp. 150-165.

Esen, Murat, Mehmet Sukru Bellibas y Sedat Gumus (2020), "The evolution of leadership research in higher education for two decades (1995-2014): a bibliometric and content analysis", International Journal of Leadership in Education, vol. 23, núm. 3, pp. 259-273.

Feixas, Mónica, María-Jesús Martínez-Usarralde y Ramón López-Martín (2018), "Do teaching innovation projects make a difference? Assessing the impact of small-scale funding", Tertiary Education and Management, vol. 24, pp. 267-283.

Fossatti, Paulo, Francisco Ganga Contreras y Hildegard Jung (2017), "Reflexiones en torno a la gobernanza universitaria: Una mirada desde Latinoamérica", Revista Espacios, vol. 38, núm. 35, pp. 14-26.

Fraser, Sharon (2019), "Understanding innovative teaching practice in higher education: a framework for reflection", Higher Education Research \& Development, vol. 38, núm. 7, pp. 1371-1385.

Ganga-Contreras, Francisco, Emilio Rodríguez-Ponce, Erwin Navarrete y Liliana Pedraja-Rejas (2018), "Relevancia del liderazgo en el gobierno de las universidades iberoamericanas", Interciencia, vol. 43, núm. 3, pp. 160-167.

García Guadilla, Carmen (2007), "Financiamiento de la educación superior en América Latina", Porto Alegre, vol. 9, núm. 17, pp. 50-101.

Garwe, Evelyn Chiyevo (2014), "The effect of institutional leadership on quality of higher education provision", Research in Higher Education fournal, vol. 22, pp. 1-10.

Gaus, Nurdiana, Muhammad Basri, Husni Thamrin y Fajar Utama Ritonga (2020), "Understanding the nature and practice of leadership in higher education: a phenomenological approach", International fournal of Leadership in Education, pp. 1-19.

Getz, Isaac y Alan Robinson (2003), "Innovate or die: is that a fact?", Creativity and Innovation Management, vol. 12, núm. 3, pp. 130-136. 
Gibbs, Graham, Christopher Knapper y Sergio Piccinin (2008), "Disciplinary and contextually appropriate approaches to leadership of teaching in researchintensive academic departments in higher education", Higher Education Quarterly, vol. 62, núm. 4, pp. 416-436.

Gosling, Jonathan, Richard Bolden y Georgy Petrov (2009), "Distributed leadership in higher education: what does it accomplish?", Leadership, vol. 5, pp. 299-310.

Gumusluoglu, Lale y Arzu Ilsev (2009), "Transformational leadership, creativity, and organizational innovation", Fournal of Business Research, vol. 62, núm. 4, pp. 461-473.

Gunn, Vicky y Anna Fisk (2013), Considering teaching excellence in higher education: 2007-2013: a literature review since the CHERI Report 2007, York, UK, Higher Education Academy.

Harkin, Damien y Annah Healy (2013), "Redefining \& leading the academic discipline in Australian universities", Australian Universities' Review, vol. 55, núm. 2, pp. 80-92.

Harris, Alma (2007), "Distributed leadership: conceptual confusion and empirical reticence", International fournal of Leadership in Education, vol. 10, núm. 3, pp. 315-325.

Harris, Alma y John DeFlaminis (2016), "Distributed leadership in practice: evidence, misconceptions and possibilities", Management in Education, vol. 30, núm. 4, pp. 141-146.

Harvey, Marina y Sandra Jones (2020), "Enabling leadership capacity for higher education scholarship in learning and teaching (SOTL) through action research", Educational Action Research, vol. 29, núm. 2, pp. 173-190.

Hattam, Sarah y Tanya Weiler (2021), "'Every single student counts': leadership of professional development underpinned by social justice for sessional staff in a South Australian university", Professional Development in Education, vol. 47, núm. 1, pp. 128-140.

Hemmati, Reza y Arefeh Mahdie (2020), "Iranian PhD students' experiences of their learning environment and scholarly condition: a grounded theory study," Studies in Higher Education, vol. 45, núm. 1, pp. 187-208.

Ibrahim, Mohammed Mansur y Muesser Nat (2019), "Blended learning motivation model for instructors in higher education institutions", International fournal of Educational Technology in Higher Education, vol. 16, pp. $1-21$.

Igwe, Paul A., Dieu Hack-Polay, John Mendy, Ted Fuller y Deborah Lock (2019), "Improving higher education standards through reengineering in West African universities - A case study of Nigeria", Studies in Higher Education, pp. 1-14.

Jaime Cuadros, María del Pilar, María P. Cáceres Reche y Francisco Hinojo Lucena (2018), "'Analysis of leadership styles developed by teachers and administrators in technical-technological programs: the case of the Cooperative University of Colombia'”, International Fournal of Leadership in Education, vol. 21, núm. 1, pp. 1-16. Jones, Sandra y Marina Harvey (2017), "A distributed leadership change process model for higher education", Fournal of Higher Education Policy and Management, vol. 39, núm. 2, pp. 126-139.

Jones, Sandra, Kevin Ryland, Marina Harvey, Geraldine Lefoe, Annette Schneider y Ann Applebee (2012), Lessons learnt: identifying synergies in distributed leadership projects, Melbourne, Australian Government Office for Learning and Teaching.

Jones, Sandra, Marina Harvey, Jillian Hamilton, John Bevacqua, Kathy Egea y Jo McKenzie (2017), "Demonstrating the impact of a distributed leadership approach in higher education", fournal of Higher Education Policy and Management, vol. 39, núm. 2, pp. 197-211.

Joslyn, Erica (2018), "Distributed leadership in HE: a scaffold for cultural cloning and implications for BME academic leaders", Management in Education, vol. 32, núm. 4, pp. 185-191.

Jyoti, Jeevan y Sonia Bhau (2016), "Empirical investigation of moderating and mediating variables in between transformational leadership and related outcomes", International Fournal of Educational Management, vol. 30, núm. 6, pp. 1123-1149.

Keppell, Mike, Carolyn O’Dwyer, Betsy Lyon y Merilyn Childs (2010), "Transforming distance education curricula through distributive leadership", $A L T-\mathcal{F}$, vol. 18, núm. 3, pp. 165-178. 
Kivistö, Jussi y Vuokko Kohtamäki (2016), "Does performance-based funding work? reviewing the impacts of performance-based funding on higher education institutions", en Rosalind M. O. Pritchard, Attila Pausits y James Williams (eds.), Positioning Higher Education Institutions from Here to There, Rotterdam, Sense Publishers, pp. 215-226.

Klein, Julie T. y Holly J. Falk-Krzesinski (2017), "Interdisciplinary and collaborative work: framing promotion and tenure practices and policies", Research Policy, vol. 46, núm. 6, pp. 1055-1061.

Koeslag-Kreunen, Mieke, Piet Van den Bossche, Marcel R. Van der Klink y Wim H. Gijselaers (2021), "Vertical or shared? When leadership supports team learning for educational change", Higher Education, vol. 82, pp. 19-37.

Kosunen, Sonja (2018), "Access to higher education in Finland: emerging processes of hidden privatization", Nordic Gournal of Studies in Educational Policy, vol. 4, núm. 2, pp. 67-77.

Kouzes, James y Barry Posner (2019), Leadership in higher education: practices that make a difference, California, BerrettKoehler Publishers.

Labraña, Julio y Raf Vanderstraeten (2020), "Functional differentiation and university expansion in Chile", Social and Education History, vol. 9, núm. 3, pp. 252-274.

Le, Phong Ba y Hui Lei (2019), "Determinants of innovation capability: the roles of transformational leadership, knowledge sharing and perceived organizational support", fournal of Knowledge Management, vol. 23, núm. 3 , pp. 527-547.

Leach, Linda (2013), "Participation and equity in higher education. Are we going back to the future?", Oxford Review of Education, vol. 39, núm. 2, pp. 267-286.

Lodders, Nanda y Frans Meijers (2017), "Collective learning, transformational leadership and new forms of careers guidance in universities", British fournal of Guidance E̊ Counselling, vol. 45, núm. 5, pp. 532-546.

López Martin, Ramón (2017), "Hacia una innovación docente de calidad en la educación superior. Claves para la reflexión", Foro Educacional, vol. 28, pp. 11-28.

Majeed, Nauman, T. Ramayah, Norizah Mustamil,
Mohammad Nazri y Samia Jamshed (2017), "Transformational leadership and organizational citizenship behavior: Modeling emotional intelligence as mediator", Management \& Marketing. Challenges for the Knowledge Society, vol. 12, núm. 4, pp. 571-590.

Marshall, Stephen (2010), "Change, technology and higher education: are universities capable of organisational change?", ALT-f, vol. 18, núm. 3, pp. 179-192.

Martin, Elaine, Keith Trigwell, Michael Prosser y Paul Ramsden (2003), "Variation in the experience of leadership of teaching in higher education", Studies in Higher Education, vol. 28, núm. 3, pp. 247-259.

McInnis, Craig, Paul Ramsden y Don Maconachie (2012), A handbook for executive leadership of learning and teaching in higher education, Canberra, Australian Government Office for Learning and Teaching.

Mendoza, Marlena L. (2015), "Innovation across types of organization: a meta-analysis", Suma de Negocios, vol. 6, núm. 13, pp. 108-113.

Middlehurst, Robin (2008), "Not enough science or not enough learning? Exploring the gaps between leadership theory and practice", Higher Education Quarterly, vol. 62, núm. 4, pp. 322-339.

Middlehurst, Robin y Lewis Elton (1992), "Leadership and management in higher education", Studies in Higher Education, vol. 17, núm. 3, pp. 251-264.

Molesworth, Mike, Elizabeth Nixon y Richard Scullion (2009), "Having, being and higher education. The marketisation of the university and the transformation of the student into consumer", Teaching in Higher Education, vol. 14, núm. 3, pp. 277-287.

Moolenaar, Nienke, Alan Daly y Peter Sleegers (2010), "Occupying the principal position: examining relationships between transformational leadership, social network position, and schools' innovative climate", Educational Administration Quarterly, vol. 46, núm. 5, pp. 623-670.

Mumford, Michael D., Ginamarie M. Scott, Blaine Gaddis y Jill M. Strange (2002), "Leading creative people: orchestrating expertise and relationships", The Leadership Quarterly, vol. 13, núm. 6, pp. 705-750. 
Navarro-Corona, Claudia (2016), “Consideraciones teóricas sobre el concepto de liderazgo y su aplicación en la investigación educativa", Revista Educación, vol. 40, núm. 1, pp. 53-66.

Newton, Jethro (2002), "Barriers to effective quality management and leadership: case study of two academic departments", Higher Education, vol. 44, pp. 185-212.

Ng'ambi, Dick y Vivienne Bozalek (2013), 'Leveraging informal leadership in higher education institutions: a case of diffusion of emerging technologies in a southern context", British Fournal of Educational Technology, vol. 44, núm. 6, pp. 940-950.

Nizami, Rahila y Khalid Rashid (2018), "Academic roles of educational leaders for quality learning environment in the public and private universities at Lahore", Annals of King Edward Medical University, vol. 24, núm. 4, pp. 948-952.

Ordorika Sacristán, Imanol, Jorge Martínez Stack y Rosa M. Ramírez Martínez (2011), "La transformación de las formas de gobierno en el sistema universitario público mexicano: una asignatura pendiente", Revista de la Educación Superior, vol. 40, núm. 160, pp. 51-68.

Ordorika, Imanol (1995), “Organización, gobierno y liderazgo universitario: una base conceptual para el análisis del cambio en la educación superior”, en Humberto Muñoz García y Roberto Rodríguez Gómez (coords.), La Universidad Mexicana a Debate, México, UNAM, pp. 161-174.

Pedraja-Rejas, Liliana, Emilio Rodríguez-Ponce y Julio Labraña (2021), “QQué sabemos de la cultura académica? Revisión del concepto en la literatura especializada en educación superior", Educação e Pesquisa (en prensa).

Pedraja-Rejas, Liliana, Giuliani Coluccio-Piñones, Andrés Bernasconi, Ítalo Marchioni-Choque y Camila Muñoz-Fritis (2019), "Cultura y estilos de liderazgo en unidades académicas: un estudio en una institución de educación superior", Utopía y Praxis Latinoamericana, vol. 24, núm. 4, pp. 25-35.

Pedraja-Rejas, Liliana, Roberto I. Vega y Jaime Riquelme Castañeda (2018), "La importancia de los estilos de liderazgo en la calidad de las unidades académicas universitarias", Opción, núm. 86, pp. 130-151.
Pepper, Coral y Susan Roberts (2016), "Valuing the leadership role of university unit coordinators", Issues in Educational Research, vol. 26, núm. 1, pp. 117-130.

Pérez, Giovanni, Lorena Guarín y Gerardo Romo (2015), "Liderazgo transformacional en los docentes universitarios desde la perspectiva estudiantil. Estudio de caso: Universidad Nacional de Colombia, sede Medellín”, Saber, Ciencia y Libertad, vol. 10, núm. 1, pp. 203-218.

Pineda, Pedro (2015), The entrepreneurial research university in Latin America: global and local models in Chile and Colombia, 1950-2015, Nueva York, Palgrave Macmillan.

Pounder, James, Peter Stoffell y Edward Choi (2018), "Transformational classroom leadership and workplace engagement", Quality Assurance in Education, vol. 26, núm. 3, pp. 333-342.

Quinlan, Kathleen (2014), "Leadership of teaching for student learning in higher education: what is needed?", Higher Education Research $\Xi$ Development, vol. 33, núm. 1, pp. 32-45.

Ramsden, Paul (1998), Learning to lead in higher education, Londres, Routledge Falmer.

Ramsden, Paul, Michael Prosser, Keith Trigwell y Elaine Martin (2007), "University teachers' experiences of academic leadership and their approaches to teaching", Learning and Instruction, vol. 17, núm. 2, pp. 140-155.

Robinson, Viviane, Claire Lloyd y Kenneth Rowe (2008), "The impact of leadership on student outcomes: an analysis of the differential effects of leadership types", Educational Administration Quarterly, vol. 44, núm. 5, pp. 635-674.

Rodríguez Pulido, Josefa, Josué Artiles Rodríguez y María V. Aguiar Perera (2015), "La gestión universitaria: dificultades en el desempeño del cargo y necesidades de formación", REDU. Revista de Docencia Universitaria, vol. 13, núm. 3, pp. 213-231.

Rodríguez-Ponce, Emilio, Liliana Pedraja-Rejas y Francisco Ganga-Contreras (2019), "Determinantes, procesos y resultados de la formación inicial de profesores de enseñanza básica y educación parvularia en Chile: una aproximación conceptual", Formación Universitaria, vol. 12, núm. 6, pp. 127-140. 
Schwab, Klaus (2016), The fourth industrial revolution, Cologny/Geneva, Suiza, World Economic Forum.

Scott, Geoff, Hamish Coates y Michelle Anderson (2008), Learning leaders in times of change: academic leadership capabilities for Australian higher education, Sydney, University of Western Sydney/Australian Council for Educational Research.

Scott, Scott y Rob Guthrie (2003), "Exploring the role of leadership in creating and fostering learning communities within Business Law higher education", The International fournal of Learning, vol. 10, núm.1.

Sewerin, Thomas y Robert Holmberg (2017), "Contextualising distributed leadership in higher education", Higher Education Research and Development, vol. 36, núm. 1, pp. 1280-1294.

Shankar Singh, Uma y Osman Sahin (2017), "Measuring the university competence comparatively for nurturing future leaders", Scholars Fournal of Economics, Business and Management, vol. 4, núm. 10, pp. 669-680.

Siddique, Anam, Hassan Danial Aslam, Mannan Khan y Urooj Fatima (2011), "Impact of academic leadership on faculty's motivation and organizational effectiveness in higher education system", International fournal of Academic Research, vol. 3, núm. 2, pp. 730-737.

Spillane, James, Richard Halverson y John Diamond (2004), "Towards a theory of leadership practice: a distributed perspective", Fournal of Curriculum Studies, vol. 36, núm. 1, pp. 3-34.

Steinbicker, Jochen (2011), "Zur theorie der informationsgesellschaft: ein vergleich der ansätze von Peter Drucker, Daniel Bell und Manuel Castells", Wiesbaden, VS Verl.

Sutton, Kimberly K. y Josh DeSantis (2017), "Beyond change blindness: embracing the technology revolution in higher education", Innovations in Education and Teaching International, vol. 54, núm. 3, pp. 223-228.

Thornton, Kate, Jo Walton, Marc Wilson y Liz Jones (2018), "Middle leadership roles in universities: Holy Grail or poisoned chalice", Journal of Higher Education Policy and Management, vol. 40, núm. 3, pp. 208-223.
Trivellas, Panagiotis y Dimitra Dargenidou (2009), "Leadership and service quality in higher education: the case of the Technological Educational Institute of Larissa", International Journal of Quality and Service Sciences, vol. 1, núm. 3, pp. 294-310.

Trow, Martin (2007), "Reflections on the transition from elite to mass to universal access: forms and phases of higher education in modern societies since WWII", en James J. F. Forest y Philip G. Altbach (eds.), International Handbook of Higher Education: Springer International Handbooks of Education, Dordrecht, Springer, pp. 243-280.

Tünnerman, Carlos (2003), La universidad latinoamericana ante los retos del siglo XXI, México, Unión de Universidades de América Latina.

Udin, Udin, Sri Handayani, Ahyar Yuniawan y Edy Rahardja (2019), "Leadership styles and communication skills at Indonesian higher education: patterns, influences, and applications for organization", Organizations and Markets in Emerging Economies, vol. 19, pp. 111-131.

Velázquez Hernández, Juan y Gladys Hernández Romero (2019), "Influencia del liderazgo docente en la motivación de universitarios", Revista Internacional de Investigación e Innovación Educativa, núm. 13, pp. 1-16.

Vuori, Johanna (2019), "Distributed leadership in the construction of a new higher education campus and community", Educational Management Administration $\mathcal{E}^{\circ}$ Leadership, vol. 47, núm. 2, pp. 224-240.

Walker, Greg (2019), "Higher education leadership and management as "practical reasonableness": a phronetic approach to higher education research", Theory and Method in Higher Education Research, vol. 5, pp. 73-90.

Woods, Philip (2016), "Authority, power and distributed leadership", Management in Education, vol. 30, núm. 4, pp. 155-160.

World Bank (2003), Lifelong learning in the global knowledge economy: challenges for developing countries: a World Bank report, Washington D.C., The International Bank for Reconstruction and Development/The World Bank.

Youngs, Howard (2017), "A critical exploration of collaborative and distributed leadership in higher education: developing an alternative ontology through 
leadership-as-practice", Fournal of Higher Education Policy and Management, vol. 39, núm. 2, pp. 140-154.

Zepke, Nick (2015), "Student engagement research: thinking beyond the mainstream", Higher Education Research E̊ Development, vol. 34, núm. 6, pp. 1311-1323.
Zuraik, Abdelrahman y Louise Kelly (2019), "The role of CEO transformational leadership and innovation climate in exploration and exploitation", European Journal of Innovation Management, vol. 22, núm. 1, pp. 84104.

\section{Cómo citar este artículo:}

Rodríguez-Ponce, Emilio, Liliana Pedraja-Rejas y Julio Labraña (2022), “Liderazgo en el gobierno universitario e innovaciones en la docencia: una revisión de la literatura", Revista Iberoamericana de Educación Superior (RIES), vol. XIII, núm. 36, pp. 102-123, DOI: https://doi.org/10.22201/iisue.20072872e.2022.36.1186 [Consulta: fecha de última consulta]. 